\title{
Erratum to: Evolution of educational inequalities in mortality among young adults in an urban setting
}

\author{
Hannelore De Grande • Patrick Deboosere • \\ Hadewijch Vandenheede
}

Published online: 13 February 2015

(C) Swiss School of Public Health 2015

\section{Erratum to: Int J Public Health (2013) 58:825-835 \\ DOI 10.1007/s00038-013-0478-x}

Due to an error in the calculation of the standard population, the age-standardised mortality rates (ASMRs) in Table 2 were underestimated.

The interpretation of the results does not change.

We hereby provide the corrections for the text and the revised table with the corrected ASMRs, directly standardised to the Brussels population.

\section{Corrected text:}

P 828: The ASMR for men is 145.1 [135.1-155.1] (per $100,000 \mathrm{PY}$ ) in the period 1991-1996, compared to 86.2 [78.6-93.8] in 2001-2006 (Table 2).

P 829: First of all, mortality rates do not differ as much between the two periods as they do among men: from 61.4 [54.9-68.0] to 40.2 [35.0-45.4].

The online version of the original article can be found under doi:10.1007/s00038-013-0478-x.

H. De Grande $(\bowtie) \cdot$ P. Deboosere · H. Vandenheede Interface Demography, Department of Sociology, Vrije Universiteit Brussel, Pleinlaan 5, 1050 Brussels (Elsene), Belgium

e-mail: hannelore.de.grande@vub.ac.be

H. Vandenheede

Research Department of Epidemiology and Public Health, Institute of Epidemiology and Health Care, University College London, London, UK 


\section{Corrected table:}

Table 2 Age-standardised all-cause mortality rates (ASMRs) and the relative index of inequality (RII), separately for men and women and period, according to educational level of the Brussels-Capital Region (Belgium)

\begin{tabular}{|c|c|c|c|c|c|c|}
\hline & \multicolumn{3}{|c|}{$1991-1996$} & \multicolumn{3}{|c|}{ 2001-2006 } \\
\hline & $P Y^{\mathrm{a}}$ & ASMR & Deaths & $\mathrm{PY}^{\mathrm{a}}$ & ASMR & Deaths \\
\hline \multicolumn{7}{|l|}{ Men } \\
\hline No/primary & 79,889 & 231.6 [193.4-269.9] & 182 & 35,091 & $120.5[82.8-158.3]$ & 43 \\
\hline Lower secondary & 71,452 & $167.8[137.2-198.3]$ & 118 & 99,077 & $105.1[84.1-126.2]$ & 99 \\
\hline Higher secondary & 185,437 & $140.3[120.7-160.0]$ & 230 & 157,725 & $86.3[70.8-101.8]$ & 129 \\
\hline Higher education & 135,911 & $99.7[77.4-122.1]$ & 125 & 192,331 & $44.2[32.7-55.6]$ & 81 \\
\hline Missing & 98,961 & $159.3[134.0-184.6]$ & 165 & 91,735 & $152.4[127.1-177.7]$ & 143 \\
\hline \multirow[t]{2}{*}{ Total } & 571,650 & $145.1[135.1-155.1]$ & 820 & 575,959 & $86.2[78.6-93.8]$ & 495 \\
\hline & & \multicolumn{3}{|c|}{$\mathrm{RII}=3.07 * * *[2.33-4.06]$} & \multicolumn{2}{|c|}{$\mathrm{RII}=4.09 * * *[2.78-6.03]$} \\
\hline \multicolumn{7}{|l|}{ Women } \\
\hline No/primary & 82,419 & $85.4[60.1-110.7]$ & 67 & 36,938 & 73.5 [46.0-101.1] & 29 \\
\hline Lower secondary & 59,269 & $67.3[45.9-88.8]$ & 39 & 87,416 & $32.2[19.7-44.7]$ & 27 \\
\hline Higher secondary & 180,527 & $69.1[55.2-83.1]$ & 108 & 147,801 & $34.2[24.1-44.4]$ & 47 \\
\hline Higher education & 159,147 & $41.0[26.9-55.2]$ & 55 & 225,089 & $32.2[24.3-40.0]$ & 76 \\
\hline Missing & 92,318 & $81.2[62.8-99.6]$ & 79 & 81,607 & $61.6[44.4-78.8]$ & 50 \\
\hline Total & 573,680 & $61.4[54.9-68.0]$ & 348 & 578,851 & $40.2[35.0-45.4]$ & 229 \\
\hline \multicolumn{7}{|c|}{ RII not relevant ${ }^{b}$} \\
\hline
\end{tabular}

Standard Population: Brussels' population 2001 Census

Source: Belgian Census and Death Certificates of 1991-1996 and 2001-2006

*** Significant at the 0.001-level

${ }^{\text {a }}$ Person years

b As there is no linear relationship between education and mortality among women, the calculation of an RII is not relevant 